\title{
INCLUSIONS OF HARDY ORLICZ SPACES
}

\author{
ROSHDI KHALIL \\ Department of Mathematics \\ The University of Michigan \\ Ann Arbor, Michigan 48109 U.S.A. \\ (Received September 9, 1985 and in revised form February 24, 1986)
}

ABSTRACT. Let $\phi$ be a continuous positive increasing function defined on $[0, \infty)$ such that $\phi(x+y) \leq \phi(x)+\phi(y)$ and $\phi(0)=0$. The Hardy-Orlicz space generated by $\phi$ is denoted by $H(\phi)$. In this paper, we prove that for $\phi \neq \psi$, if $H(\phi)=H(\psi)$ as sets, then $H(\phi)=H(\psi)$ as topological vector spaces. Some other results are given.

KEY WORDS AND PHRASES. Modulus function, Orlicz spaces.

1980 AMS SUBJECT CLASSIFICATION CODE. $30 \mathrm{G} 99$.

1. INTRODUCTION.

Let $\phi:[0, \infty) \longrightarrow[0, \infty)$ be a continuous increasing function such that $\phi(x+y) \leq$ $\phi(x)+\phi(y)$ and $\phi(0)=0$. Let $\mathrm{T}$ be the unit circle, and $\mathrm{m}$ be the Lebesgue measure on $T$. A complex valued measurable function $f^{\cdot}$ defined on $T$ is called $\phi$-integrable if $\int_{I} \phi|f(t)| d m(t)<\infty$. The space of all $\phi$-integrable functions on $T$ will be denoted by $L(\phi)$. This space was first introduced by Orlicz, [8]. Subsequent papers were written to study different aspects of $L(\phi)$. Examples of these papers are Cater, [4], Gramsch, [5] and Pallashke [9].

In [6] and [7], Lesniewicz introduced the so called Hardy-Orlicz spaces $H(\phi)$ for a given such function $\phi$. The space $H(\phi)$ was defined to be the space of all functions $f \in L(\phi)$ such that $f$ is the radial limit of some function $g$ analytic in the open unit disc and belongs to the Nevalinna class $N$. The relation between different $H(\phi)-$ spaces was studied by Deeb, Khalil and Marzug [3]. In this paper, we show that the inclusion map between two $H(\phi)$-spaces is always continuous. Some other results are given. It should be remarked that in the work of Lesniewicz, [6], [7] and many other authors, $\phi$ is assumed to be a $\phi$-convex function. In this paper it is not assumed so.

2. PRELIMINARIES AND NOTATIONS.
A function $\phi:[0, \infty) \longrightarrow[0, \infty)$ is called a modulus function if

(i) $\phi$ is continuous and increasing

(ii) $\phi(x)=0$ if and only if $x=0$

(iii) $\phi(x+y) \leq \phi(x)+\phi(y)$.

The functions $\phi(x)=x^{p}, 0<p \leq 1$ and $\phi(x)=\ln (1+x)$ are examples of modulus functions. Further, if $\phi_{1}$ and $\phi_{2}$ are modulus functions, then $\phi_{1}+\phi_{2}$ and $\phi_{1} \phi_{2}$ 
are modulus functions. Further, $\psi=\frac{\phi}{1+\phi}$ is a modulus function if $\phi$ is.

Let $T=\{z:|z|=1\}, \Delta=\{z:|z|<1\}$. The space of analytic functions on $\Delta$ is denoted by $H(\Delta)$. Let $H^{+}(\Delta)=\left\{f \in H(\Delta): \lim _{\mathbf{r} \rightarrow 1} f\left(\mathrm{re}^{\mathrm{i} \theta}\right)\right.$ exists a.e. $\left.\theta\right\}$. We will consider $\mathrm{H}^{+}(\Delta)$ as a space of functions on $T$. For a given modulus function $\phi$, we define:

$$
H(\phi)=\left\{f \in H^{+}(\Delta): \sup _{0 \leq r<1} \int_{0}^{2 \pi} \phi\left|f\left(r e^{i \theta}\right)\right| d \theta=\int_{0}^{2 \pi} \phi\left|f\left(e^{i \theta}\right)\right| d \theta<\infty\right\} .
$$

The function $d: H(\phi) \times H(\phi) \longrightarrow[0, \infty), d(f, g)=\int_{0}^{2 \pi} \phi\left|f\left(e^{i \theta}\right)-g\left(e^{i \theta}\right)\right| d \theta$ defines a metric on $\mathrm{H}(\phi)$, under which $\mathrm{H}(\phi)$ becomes a topological vector space. If one assumes that $\phi|u|$ is subharmonic for $u \in H(\Delta)$, then $H i \phi)$ turns out to be complete [2]. For $f \in H(\phi)$, we write $\|f\|_{\phi}=\int_{T} \phi\left|f\left(e^{i \theta}\right)\right| d \theta$. If $\phi(x)=x^{p}, 0<p \leq 1$, then $H(\phi)=H^{p}$ and for $\phi(x)=\ln (1+x), H(\phi)=N^{+}=\left\{f \in N: \int_{T} \ln (1+|f|)<\infty\right\}$, where $N$ is the Nevalinna class.

3. $\mathrm{I}: \mathrm{H}^{1} \longrightarrow \mathrm{H}(\phi)$ IS CONTINUOUS.

In [2], it was shown that $\mathrm{H}^{1} \subseteq \mathrm{H}(\phi)$ for all modulus functions $\phi$. The authors in [3] were not able to show that the inclusion map $I: H^{1} \rightarrow H(\phi)$ is continuous. In this section we prove that $\mathrm{I}: \mathrm{H}^{1} \longrightarrow \mathrm{H}(\phi)$ is continuous. Some other related questions are discussed.

THEOREM 2.1. Let $\phi$ and $\psi$ be two modulus functions such that $\underset{x \rightarrow \infty}{\lim \frac{\phi(x)}{\psi(x)}=\lambda}$
sts. Then: exists. Then:

(i) $\mathrm{H}(\phi)=\mathrm{H}(\psi)$ if $\lambda \neq 0$ and $\lambda$ is finite

(ii) $\mathrm{H}(\phi) \subseteq \mathrm{H}(\psi)$ if $\lambda=0$

(iii) $H(\psi) \subseteq H(\phi)$ if $\lambda=\infty$.

PROOF. (i) Let $\lambda \neq 0$ be finite. Then there exists $a_{1}, b_{1}, a_{2}, b_{2} \in[0, \infty)$ such that

$$
\begin{aligned}
& \phi(x) \leq a_{1} \psi(x) \text { for } x \in\left[a_{2}, \infty\right) \cdots(*) \\
& \psi(x) \leq b_{1} \phi(x) \text { for } x \in\left[b_{2}, \infty\right) \cdots(* *) \text {. }
\end{aligned}
$$

Let $f \in H(\psi)$. Set $E\left(a_{2}\right)=\left\{t \in T:|f(t)| \geq a_{2}\right\}$. Then

$$
\begin{aligned}
\|f\|_{\phi} & =\int_{E\left(a_{2}\right)} \phi\left|f\left(e^{i \theta}\right)\right| d \theta+\int_{E}^{c}\left(a_{2}\right) \\
& \leq a_{1}\|f\|_{\psi}+\phi\left(a_{2}\right)<\infty .
\end{aligned}
$$

Hence $f \in H(\phi)$ and $H(\psi) \subseteq H(\phi)$. Similarly we show $H(\phi) \subseteq H(\psi)$. Consequently, $H(\phi)=H(\psi)$. Case (1i) and (iii) are proved similarly and details are omitted. This ends the proof.

THEOREM 2.2. Let $\lim _{x \rightarrow \infty} \frac{\phi(x)}{\psi(x)}=\lambda>0$. Then the inclusion map $\mathrm{I}: H(\phi) \longrightarrow H(\psi)$ is continuous.

PROOF. From the proof of Theorem 2.1, there exists $a, b>0$ such that $\|f\|_{\psi \leq} \leq$ $\psi(a)+b\|f\|_{\psi}$ for all $f \in H(\phi)$.

Let $f_{n} \rightarrow 0$ in $H(\phi)$. Thus the sequence $\left(f_{n}\right)$ is bounded in the metric of $H(\phi)$ and consequently bounded in $H(\psi)$. If possible let there exist a subsequence (f ${ }_{n_{h}}$ ) 
such that $\left\|f_{n_{k}}\right\| \rightarrow \alpha>0$. Since $\left\|f_{n_{k}}\right\|_{\phi} \rightarrow 0,\left(f_{n_{k}}\right.$ ) has a subsequence which converges pointwise to the zero function. With no loss of generality, we can assume that $\mathrm{f}_{\mathrm{n}_{\mathrm{k}}} \rightarrow 0$ a.e. Another application of the proof of Theorem 2.1 , yields $\psi(\mathrm{x}) \leq$ $\psi(a)+b \cdot \phi|x|$ for all $x \in[0, \infty)$. Hence

$$
\psi\left|f_{n_{k}}(t)\right| \leq \psi(a)+b \cdot \phi\left|f_{n_{k}}(t)\right| \text {. }
$$

The sequence of functions $g_{n_{k}}=\psi(a)+b \phi\left|f_{n_{k}}\right|$ converges a.e. to $\psi(a)$ and

$$
\int_{T}^{k} g_{n_{k}}(t) d t \longrightarrow \psi(a) \text {. }
$$

Consequently, by the generalized Lebesgue convergence theorem, [10], we have

$$
\lim _{n_{k}} \int_{T} \psi\left|f_{n_{k}}(t)\right| d t=\int_{T} \lim _{n_{k}} \psi\left|f_{n_{k}}(t)\right| d t=0 .
$$

This is a contradiction. Thus, the point $w=0$ is the only limit point of the bounded sequence $\left(\left\|f_{n}\right\|_{\psi}\right)$. Consequent1y, [11], the sequence $\left\|f_{n}\right\|_{\psi}$ converges to zero. Hence $\mathrm{I}: \mathrm{H}(\phi) \rightarrow \mathrm{H}(\psi)$ is continuous. This ends the proof. spaces.

COROLlARY 2.3. If $\lim _{x \rightarrow \infty} \frac{\phi(x)}{\psi(x)}=\lambda \in(0, \infty)$, then $H(\phi)=H(\psi)$ as topological vector PROOF. By Theorem 2.1, $\mathrm{H}(\phi)=\mathrm{H}(\psi)$ as sets. Theorem 2.2 implies that $\mathrm{I}: \mathrm{H}(\phi) \rightarrow$ $H(\psi)$ is an isomorphism. This ends the proof.

A linear map $\mathrm{A}: \mathrm{H}(\phi) \longrightarrow \mathrm{H}(\psi)$ is called metrically bounded if $\|\mathrm{Af}\|_{\psi} \leq \lambda\|\mathrm{f}\|_{\phi}$ for all $\mathrm{f} \in \mathrm{H}(\phi)$ and some $\lambda>0$. Clearly every metrically bounded map is cont nuous. The converse need not be true. However, for the inclusion map, we have the following:

THEOREM 2.4. Let $\phi$ be any modulus function. Then there exists $\lambda>0$ such that for all $f \in H^{1},\|f\|_{1} \geq 1,\|f\|_{\phi} \leq \lambda\|f\|_{1}$.

PROOF. It is know, [2] (and easy to show) that $H^{1} \subseteq H(\phi)$ for all modulus functions $\phi$. If $f \in H^{1}$ and $\|f\|_{1}=1$, then using the argument in Theorem 2.1, we have $\|f\|_{\phi} \leq \lambda\|f\|_{1}$.

Let $f \in \mathrm{H}^{1},\|f\|_{1}>1$. Then there exists $0<\alpha<1$ such that $\|\alpha f\|_{1}=1$. Since $\alpha<1$, there exists a natural number $n$ such that $\frac{1}{n+1} \leq \alpha \leq \frac{1}{n}$. Hence

$$
\|\alpha f\|_{\phi} \leq \lambda\|\alpha f\|_{1}=\lambda \alpha\|f\|_{1} \text {. }
$$

But $\left\|\frac{1}{n+1} \mathrm{f}\right\|_{\phi} \leq\|\alpha \mathrm{f}\|_{\phi}$, and $\left\|\frac{1}{\mathrm{k}} \mathrm{f}\right\|_{\phi} \geq \frac{1}{\mathrm{k}}\|\mathrm{f}\|_{\phi}$ for any modulus function $\phi$. It follows that:

$$
\frac{1}{n+1}\|f\|_{\phi} \leq \lambda \cdot \alpha\|f\|_{1} \leq \frac{\lambda}{n}\|f\|_{1},
$$

and consequently

This ends the proof.

$$
\|f\|_{\phi} \leq \lambda \frac{n+1}{n}\|f\|_{1} \leq 2 \lambda\|f\|_{1} .
$$

THEOREM 2.5. Let $\phi$ be a given modulus function such that $H^{1}=H(\phi)$. If metric and topological bounded sets coincide in $H(\phi)$, then $\|f\|_{1} \leq \lambda\|f\|_{\lambda}$ for all $f \in H(\phi)$, $\|f\|_{\phi} \leq 1$ for some $\lambda>0$. 
PROOF. Applying Corollary 2.3, I: $\mathrm{H}(\phi) \rightarrow \mathrm{H}^{1}$ is an isomorphism of topological vector spaces. If possible, let $\|f\|_{1} \leq \lambda\|f\|_{\phi}$ be not true on the unit sphere of $H(\phi)$. Then, for each $n$, there exists $f_{n} \in H(\phi),\left\|f_{n}\right\|_{\phi}=1$ such that

$$
\left\|f_{n}\right\|_{1} \geq n\left\|f_{n}\right\|_{\phi}=n
$$

Consider the sequence $\frac{f_{n}}{n}=g_{n}$. By the assumption on bounded sets of $H(\phi)$, we have, [12], $g_{n} \rightarrow 0$ in $H(\phi)$. But $\left\|g_{n}\right\|_{1}=\left\|\frac{f_{n}}{n}\right\|_{1} \geq 1$ for all $n$. Th1s contradicts the continuity of the identity map $\mathrm{I}: \mathrm{H}(\phi) \rightarrow \mathrm{H}^{1}$. Hence there exists $\lambda>0$ such that:

$$
\|f\|_{1} \leq \lambda\|f\|_{\phi} \quad \cdots \quad(*)
$$

for all $f \in H(\phi),\|f\|_{\phi}=1$.

Let $f \in H(\phi),\|f\|_{\phi}<1$. Consider the map $K$ : $[0, \infty) \rightarrow[0, \infty), K(t)=\|t f\|_{\phi}$. It can be easily seen that $K$ is continuous. Hence there exists a $>1$ such that $K(a)=1$. Thus for every $f \in H(\phi),\|f\|_{\phi}<1$, we can find $a>1$ such that $\|$ af $\|_{\phi}=1$. Hence, from equation $(*)$, we get:

$$
\| \text { af }\left\|_{1} \leq \lambda\right\| \text { af }\left\|_{\phi} \leq 2 a \lambda\right\| f \|_{\phi} .
$$

Consequently, $\|f\|_{1} \leq 2 \lambda\|f\|_{\phi}$. This end the proof.

\section{FURTHER RESULTS}

The concept of metrically bounded linear operator was introduced in Section 3. A linear map A: $\mathrm{H}(\phi) \rightarrow \mathrm{H}(\psi)$ is called metrically bounded if there exists $\lambda \in(0, \infty)$ such that $\|$ Af $\left\|_{\psi} \leq \lambda\right\| f \|_{\phi}$. In general, a continuous linear map need not be metrically bounded. In this section we prove a result which is a generalization of Theorem 3.1 in [3].

THEQREM 4.1. Let $\phi$ and $\psi$ be any two modules functions. Then the following are equivalent:

(i) $\frac{\lim }{x \rightarrow 0} \frac{\phi(x)}{\psi(x)}=\delta, \frac{\lim }{x \rightarrow \infty} \frac{\phi(x)}{\psi(x)}=\varepsilon$, for some $\varepsilon, \delta \in(0, \infty)$.

(i1) $\mathrm{H}(\phi)=\mathrm{H}(\psi)$, and the identity map $\mathrm{I}$ is metrically bounded.

PROOF. (i) $\rightarrow(i i)$. From the assumption in (i), one can choose a and $b$ in $(0, \infty)$ such that

$$
\begin{aligned}
& \frac{\phi(x)}{\psi(x)} \geq r \text { on }[0, a] \\
& \frac{\phi(x)}{\psi(x)} \geq s \text { on }(b, \infty)
\end{aligned}
$$

for some $r, s \in(0, \infty)$. Theorem 3.2 implies that $H(\phi)=H(\psi)$.

Let $f \in H(\phi)$. Consider the following sets: 


$$
\begin{aligned}
& E(a)=\left\{t: 0 \leq\left|f\left(e^{i t}\right)\right|<a\right\} \\
& E(b)=\left\{t:\left|f\left(e^{i t}\right)\right|>b\right\} \\
& E(a, b)=\left\{t: a \leq \mid f\left(e^{i t}\right) \leq b\right\} .
\end{aligned}
$$

Then:

$$
\begin{aligned}
\|f\|_{\psi} & =\int_{E(a)} \psi\left|f\left(e^{i t}\right)\right| d t+\int_{E(a, b)} \psi\left|f\left(e^{i t}\right)\right| d t+\int_{E(b)} \psi\left|f\left(e^{i t}\right)\right| d t \\
& \leq \frac{1}{r}\|f\|_{\phi}+\int_{E(a, b)} \psi\left|f\left(e^{i t}\right)\right| d t+\frac{1}{s}\|f\|_{\phi} .
\end{aligned}
$$

On the closed interval $[a, b]$, the continuity of $\frac{\phi(x)}{\psi(x)}$ implies the existence of $\lambda>0$ such that $\psi(x) \leq \lambda \phi(x)$. Hence

$$
\int_{E(a, b)} \psi\left|f\left(e^{i t}\right)\right| d t \leq \frac{1}{\lambda}\|f\|_{\phi} .
$$

Thus, $\|f\|_{\psi} \leq \beta\|f\|_{\phi}$ where $\beta=\max \left(\frac{1}{r}, \frac{1}{s}, \frac{1}{\lambda}\right)$. In a similar way one can show that $\|f\|_{\phi} \leq \gamma\|E\|_{\phi}$ for all $f \in H(\phi)=H(\psi)$. Hence the identity map is metrically bounded.

Conversely, (ii) $\rightarrow(i)$. Assume $H(\phi)=H(\psi)$ and $I: H(\phi) \leftrightarrow H(\psi)$ is metrically bounded. Then there exists $\alpha$ and $\beta$ in $(0, \infty)$ such that

$$
\|f\|_{\phi} \leq \alpha\|f\|_{\psi} \leq\|f\|_{\phi} \text {. }
$$

Hence $\frac{\alpha}{B} \leq \frac{\|f\|_{\phi}}{\|f\|_{\psi}} \leq \alpha$ for all $f \in H(\phi)=H(\psi)$. Consider the function $f(z)=x z$ for $z=e^{i t}, x \in(0, \infty)$. Then

$$
\|f\|_{\phi}=\phi(x) \text { and }\|f\|_{\psi}=\psi(x) .
$$

Consequently $\frac{\alpha}{\beta} \leq \frac{\phi(x)}{\psi(x)} \leq \alpha$. Since $\alpha, \beta \in(0, \infty)$, (i) then follows. This end the proof.

ACKNOWLEDGEMENT. This work was done while the author was a visiting Professor at the University of Michigan. The author would also like to thank the Department of Mathematics for their warm hospitality.

\section{REFERENCES}

1. AlEKSANDROV, A.B. Essays On No Locally Convex Hardy Classes. Lecture Notes In Math. $864(1-90)$.

2. DEEB, W. and MARZUQ, M. $H(\phi)$-Spaces. To Appear In Bull. Canad. Math. Soc.

3. DEEB, W. KHALIL, R. and MARZUQ, M. Isometric Multiplication Of Hardy-Orlicz Spaces. To Appear In Bu11. Aust. Math. Soc:

4. CATER, S. Continuous Linear Functionals On Certaln Topological Vector Spaces. Pac. J. Math. 13(1963) 65-71.

5. GRAMSCH, B. Die Klasse Der Metrischen Linearen Raume L( ) . Math. Ann. 171(1967) 61-78.

6. LESNIEWICZ, R. On Hardy-Orlicz Spaces I, Commt. Math. 15(1971) 3-56. 
7. LESNIEWICZ, R. On Linear Functionals In Hardy-Orlicz Spaces I, Stud. Math. 46(1973) $53-77$.

8. ORLICZ, w. On Spaces of $\phi$-integrable Functions. Proc. Intern. Symp. On Linear Spaces. Hebrew Univ. Jerusalem, (1960) 357-365.

9. PALLASHKE, D. The Compact Endomorphisms of The Metric Linear Spaces L( ).

Studia Math. XLVII (1973).

10. ROYDEN, H.L., Macmillan Company New York, 1963.

11. WILANSKY, A. Topology For Analysis, John Wiley, 1970.

12. WILANSKY, A. Modern Methods In Topological Vector Spaces. McGraw Hill. 1978. 


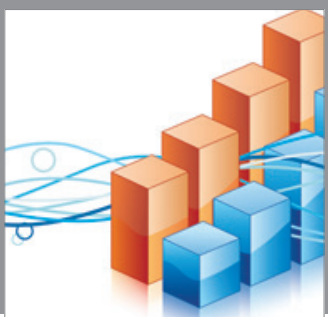

Advances in

Operations Research

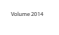

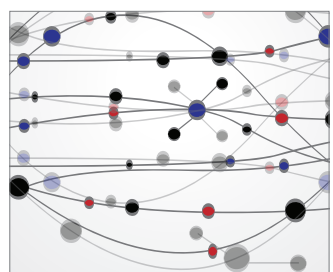

\section{The Scientific} World Journal
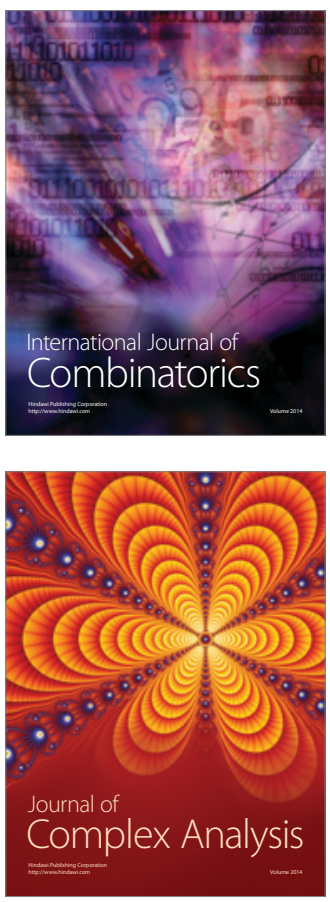

International Journal of

Mathematics and

Mathematical

Sciences
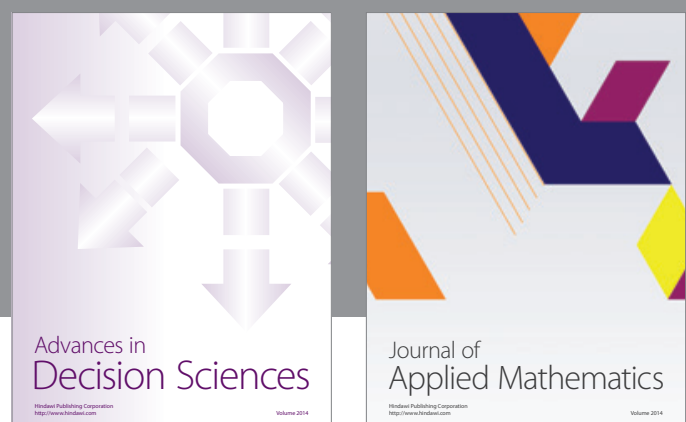

Journal of

Applied Mathematics
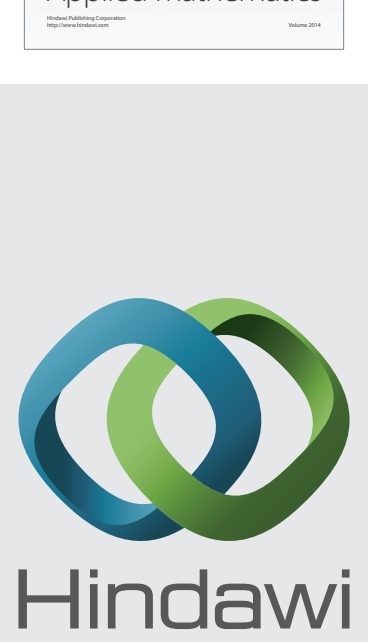

Submit your manuscripts at http://www.hindawi.com
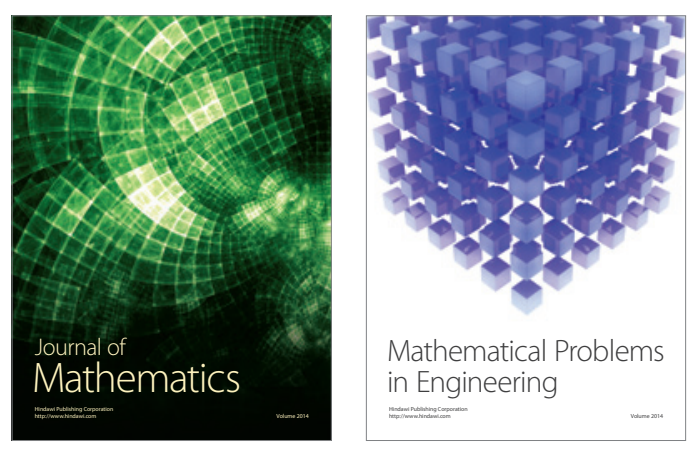

Mathematical Problems in Engineering
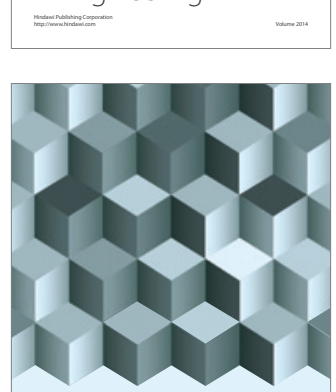

Journal of

Function Spaces
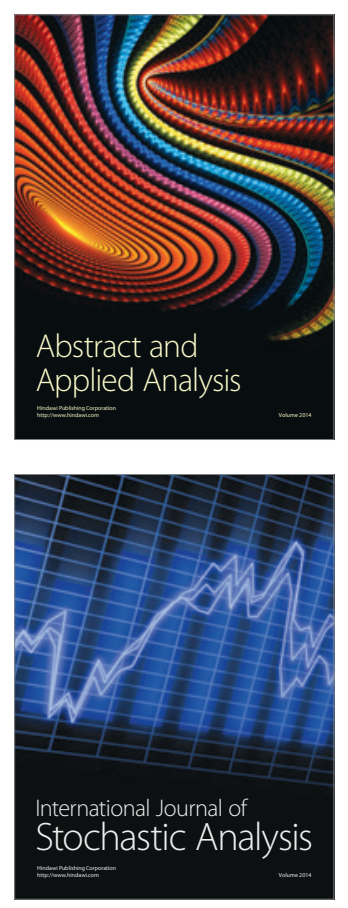

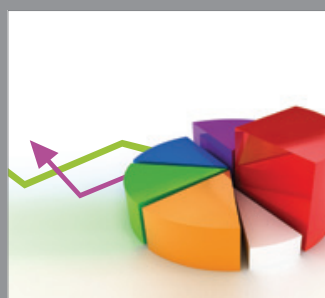

ournal of

Probability and Statistics

Promensencen
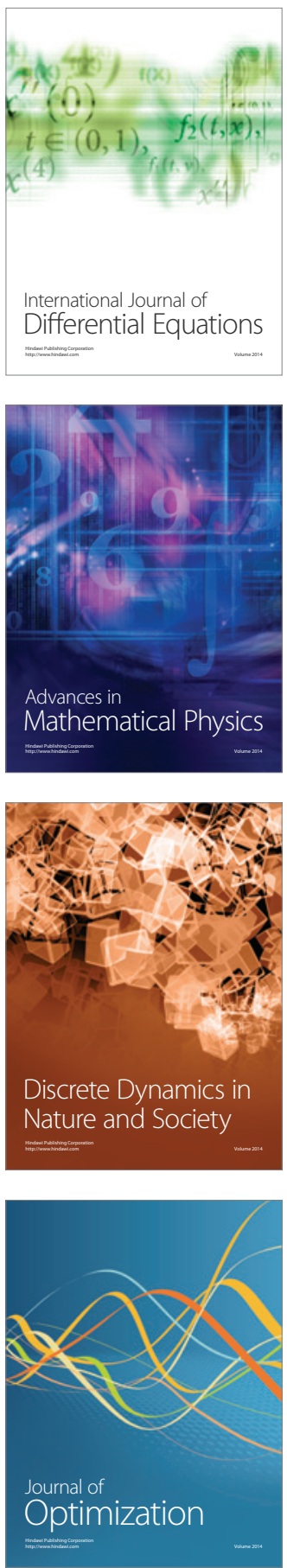